\title{
On the existence of mild solutions to the Cauchy problem for a class of fractional evolution equations
}

\author{
Jin Liang ${ }^{1 *}$, Sheng-Hua Yan ${ }^{1}$, Fang $L^{2}{ }^{2}$ and Ting-Wen Huang ${ }^{3}$
}

\footnotetext{
* Correspondence: jinliang@sjtu. edu.cn

'Department of Mathematics, Shanghai Jiao Tong University, Shanghai 200240, P. R. China Full list of author information is available at the end of the article
}

\begin{abstract}
We are concerned with the existence of mild solutions to the Cauchy problem for fractional evolution equations of neutral type with almost sectorial operators

$$
\begin{aligned}
& \frac{d^{q}}{d t^{q}}(x(t)-h(t, x(t)))=-A(x(t)-h(t, x(t)))+f(t, x(t)), \quad t>0, \\
& x(0)=x_{0}
\end{aligned}
$$

where $0<q<1$, the fractional derivative is understood in the Caputo sense, $\mathrm{A}$ is an almost sectorial operator on a complex Banach space, and $f, h$ are given functions. With the help of the theory of measure of noncompactness and a fixed point theorem of Darbo type, we establish a new existence theorem of mild solutions for the Cauchy problem above. By the way, the global attractive property of the solutions is also obtained. Moreover, we give two examples to illustrate our abstract results.
\end{abstract}

Keywords: fractional evolution equations, mild solutions, almost sectorial operators, neutral type, measure of noncompactness, global attractive

\section{Introduction}

The fractional evolution equations have received increasing attention during recent years and have been studied extensively (see, e.g., [1-13] and references therein) since they can be used to describe many phenomena arising in engineering, physics, economy, and science.

We mention that much of the previous research on the evolution equations was done provided that the operator in the linear part is the infinitesimal generator of a strongly continuous operator semigroup, an analytic semigroup, or a compact semigroup, or is a Hille-Yosida operator (see, e.g., [1-12,14,15] and references therein). On the other hand, when the operator in the linear part is an almost sectorial operator, for which the resolvent operators do not satisfy the required estimate to be a sectorial operator (see the example of almost sectorial operators which are not sectorial given by von Wahl in [16]), much less is known about the fractional evolution equations of neutral type with almost sectorial operators.

In this article, we will pay our attentions to the existence of mild solutions to the following Cauchy problem for fractional evolution equations of neutral type with almost

(c) 2012 Liang et al; licensee Springer. This is an Open Access article distributed under the terms of the Creative Commons Attribution License (http://creativecommons.org/licenses/by/2.0), which permits unrestricted use, distribution, and reproduction in any medium, provided the original work is properly cited. 
sectorial operators

$$
\begin{aligned}
& \frac{d^{q}}{d t^{q}}(x(t)-h(t, x(t)))=-A(x(t)-h(t, x(t)))+f(t, x(t)), \quad t>0, \\
& x(0)=x_{0}
\end{aligned}
$$

where $0<q<1$, the fractional derivative is understood in the Caputo sense, $A$ is an almost sectorial operator on a complex Banach space, and $f, h$ are given functions. We will use the theory of measure of noncompactness and a fixed point theorem of Darbo type to establish a new existence theorem for the problem (1.1)-(1.2). By the way, the global attractive property of the solutions are also obtained. Moreover, we give two examples to illustrate our abstract results.

This article is organized as follows: In Section 2, we state some basic concepts, notations and properties about fractional order operator and measure of noncompactness. A new existence result and the global attractive property of the solutions will be given and proved in Section 3. Finally, in Section 4, we present two concrete examples, whose physical background is statistical physics and fractional quantum mechanics (see, e.g., [12,13]).

\section{Basic concepts, notations and lemmas}

Let $X$ be a complex Banach space with norm $\|\cdot\|$ and $B(x, r)$ denote the closed ball centered at $x$ and with radius $r$. Suppose $\mathcal{M}_{X}$ denotes the family of all nonempty and bounded subsets of $X$ and subfamily consisting of all relatively compact sets is denoted by $\mathcal{N}_{X}$. As usual, for a linear operator $A$, we denote by $D(A)$ the domain of $A$, by the family $R(z ; A)=(z I-A)^{-1}, z \in \rho(A)$ of bounded linear operators the resolvent of $A$. Moreover, we denote by $L(X, X)$ the space of all bounded linear operators from Banach space $X$ to $X$ with the usual operator norm $\|\cdot\|_{L(X, X)}$, and we abbreviate this notation to $L(X)$.

Definition 2.1 [12] The fractional integral of order $q$ with the lower limit zero for a function $f \in A C[0, \infty)$ is defined as

$$
I^{q} f(t)=\frac{1}{\Gamma(q)} \int_{0}^{t}(t-s)^{q-1} f(s) d s, \quad t>0, \quad 0<q<1,
$$

provided the right side is point-wise defined on $[0, \infty)$, where $\Gamma(\cdot)$ is the gamma function.

Definition 2.2 [12] Riemann-Liouville derivative of order $q$ with the lower limit zero for a function $f \in A C[0, \infty)$ can be written as

$$
{ }^{L} D^{q} f(t)=\frac{1}{\Gamma(1-q)} \frac{d}{d t} \int_{0}^{t}(t-s)^{-q} f(s) d s, \quad t>0, \quad 0<q<1 .
$$

Definition 2.3 [12] The Caputo derivative of order $q$ for a function $f \in A C[0, \infty)$ can be written as

$$
{ }^{c} D^{q} f(t)={ }^{L} D^{q}(f(t)-f(0)), \quad t>0, \quad 0<q<1 .
$$


where ${ }^{c} D^{q}:=\frac{d^{q}}{d t^{q}}$.

Next, we recall the concept of measure of noncompactness (cf. [17]).

Definition 2.4 $\mu: \mathcal{M}_{X} \rightarrow \mathbf{R}_{+}$is said to be a measure of noncompactness in $X$ if it satisfies the following conditions:

(1) the family Ker $\mu=\left\{\Omega \in \mathcal{M}_{X} ; \mu(\Omega)=0\right\}$ is nonempty and Ker $\mu \subset \mathcal{N}_{X}$;

(2) $\Omega \subset \Omega_{0} \Rightarrow \mu(\Omega) \leq \mu\left(\Omega_{0}\right)$, for $\Omega$ and $\Omega_{0} \in \mathcal{M}_{X}$;

(3) $\mu(\operatorname{Conv}(\Omega))=\mu(\Omega)$, where $\operatorname{Conv}(\Omega)$ denotes the convex hull of $\Omega$;

(4) $\mu(\bar{\Omega})=\mu(\Omega)$, where $\bar{\Omega}$ denotes the closure of $\Omega \in \mathcal{M}_{X}$;

(5) $\mu\left(\lambda \Omega+(1-\lambda) \Omega_{0}\right) \leq \lambda \mu(\Omega)+(1-\lambda) \mu\left(\Omega_{0}\right)$, for $\lambda \in[0,1]$ and any

$\Omega, \Omega_{0} \in \mathcal{M}_{X}$

(6) If $\left\{\Omega_{n}\right\}$ is a sequence of sets from $\mathcal{M}_{X}$ such that $\Omega_{n+1} \subset \Omega_{n}$, $\bar{\Omega}_{n}=\Omega_{n}(n=1,2, \ldots)$, and if $\lim _{n \rightarrow \infty} \mu\left(\Omega_{n}\right)=0$, then the intersection $\Omega_{\infty}=\bigcap_{n=1}^{\infty} \Omega_{n}$ is nonempty.

The following is a fixed point theorem of Darbo type (see [17]).

Lemma 2.5 Let $\mathfrak{M}$ be a nonempty, bounded, closed and convex subset of a Banach space $X$, and let $H: \mathfrak{M} \rightarrow \mathfrak{M}$ be a continuous mapping. Assume that there exists a constant $k \in[0,1)$, such that

$$
\mu(H(\Omega)) \leq k \mu(\Omega)
$$

for any nonempty subset $\Omega$ of $\mathfrak{M}$. Then $H$ has a fixed point in $\mathfrak{M}$.

Let $-1<\gamma<0$, and $S_{\mu}^{0}$ with $0<\mu<\pi$ be the open sector

$$
\{z \in \mathbf{C} \backslash\{0\} ;|\arg z|<\mu\}
$$

and $S \mu$ be its closure, that is

$$
S_{\mu}=\{z \in \mathbf{C} \backslash\{0\} ;|\arg z| \leq \mu\} \cup\{0\},
$$

for more details, we refer to $[18,19]$.

As in [18], we state the concept of almost sectorial operators as follows.

Definition 2.6 Let $-1<\gamma<0$ and $0<\omega<\frac{\pi}{2}$. By $\Theta_{\omega}^{\gamma}(X)$ we denote the family of all linear closed operators $A: D(A) \subset X \rightarrow X$ which satisfy

(1) $\sigma(A) \subset S_{\omega}=\{z \in \mathbf{C} \backslash\{0\} ;|\arg z| \leq \omega\} \cup\{0\}$ and

(2) for every $\omega<\mu<\pi$ there exists a constant $C_{\mu}$ such that

$$
\|R(z ; A)\|_{L(X)} \leq C_{\mu}|z|^{\gamma}, \quad \text { for all } \quad z \in \mathbf{C} \backslash S_{\mu} .
$$

A linear operator $A$ will be called an almost sectorial operator on $X$ if $A \in \Theta_{\omega}^{\gamma}(X)$.

Remark 2.7 Let $A \in \Theta_{\omega}^{\gamma}(X)$. Then the definition implies that $0 \in \rho(A)$. 
We denote the semigroup associated with $A$ by $\{T(t)\}_{t \geq 0 \text {. For }} t \in S_{\frac{\pi}{2}-\omega}^{0}$,

$$
T(t)=e^{-t z}(A)=\frac{1}{2 \pi i} \int_{\Gamma_{\theta}} e^{-t z} R(z ; A) d z,
$$

here $\omega<\theta<\mu<\frac{\pi}{2}-|\arg t|$, forms an analytic semigroup of growth order $1+\gamma$. We have the following lemma on $T(t)$ [[19], Theorem 3.9].

Lemma 2.8 Let $A \in \Theta_{\omega}^{\gamma}(X)$ with $-1<\gamma<0$ and $0<\omega<\frac{\pi}{2}$ Then

(i) $T(t)$ is analytic in $\frac{S_{\pi}^{0}}{2}-\omega$ and

$$
\frac{d^{n}}{d t^{n}} T(t)=(-A)^{n} T(t), \quad \text { for all } \quad t \in S_{\frac{\pi}{2}-\omega}^{0} ;
$$

(ii) $T(s+t)=T(s) T(t)$ for all $s, t \in S_{\frac{\pi}{2}-\omega}^{0}$;

(ii) There exists a constant $C_{0}=C_{0}(\gamma)>0$ such that

$$
\|T(t)\|_{L(X)} \leq C_{0} t^{-\gamma-1}, \text { for all } t>0 ;
$$

(iv) The range $R(T(t))$ of $T(t)$ for each $t \in S_{\frac{\pi}{2}-\omega}^{0}$ is contained in $D\left(A^{\infty}\right)$. Particularly, for all $\alpha \in \mathbf{C}$ with $\operatorname{Re} \beta>0, R(T(t)) \subset D\left(A^{\beta}\right)$ and

$$
A^{\beta} T(t) x=\frac{1}{2 \pi i} \int_{\Gamma_{\theta}} z^{\beta} e^{-t z} R(z ; A) x d z, \quad \text { for all } \quad x \in X,
$$

and hence there exists a constant $C^{\prime}=C^{\prime}(\gamma, \beta)>0$ such that

$$
\left\|A^{\beta} T(t)\right\|_{L(X)} \leq C^{\prime} t^{-\gamma-R e \beta-1}, \text { for all } t>0
$$

(v) If $\beta>1+\gamma$, then $D\left(A^{\beta}\right) \subset \Sigma_{T}$, where $\Sigma_{T}$ is the continuity set of the semigroup $\{T$ $(t)\}_{t \geq 0}$, that is,

$$
\Sigma_{T}=\left\{x \in X ; \lim _{t \rightarrow 0 ; t>0} T(t) x=x\right\} .
$$

Clearly, we note that the condition (ii) of the Lemma 2.8 does not satisfy for $t=0$ or $s=0$. 
The relation between the resolvent operators of $A$ and the semigroup $T(t)$ is characterized by

Lemma 2.9 [[19], Theorem 3.13] Let $A \in \Theta_{\omega}^{\gamma}(X)$ with $-1<\gamma<0$ and $0<\omega<\frac{\pi}{2}$.

Then for every $\lambda \in \mathbf{C}$ with Re $\lambda>0$, one has

$$
R(\lambda ;-A)=\int_{0}^{\infty} e^{-\lambda t} T(t) d t .
$$

Now, we give the definition of mild solution to (1.1)-(1.2).

Definition 2.10 A continuous function $x:(0, T] \rightarrow X$ satisfying the equation

$$
x(t)=\mathcal{S}_{q}(t) x_{0}+h(t, x(t))+\int_{0}^{t}(t-s)^{q-1} \mathcal{P}_{q}(t-s) f(s, x(s)) d s
$$

for $t \in(0, T]$ is called a mild solution of (1.1)-(1.2), where

$$
\begin{aligned}
& \mathcal{S}_{q}(t) x=\int_{0}^{\infty} \Psi_{q}(\sigma) T\left(\sigma t^{q}\right) x d \sigma, \quad t \in S_{\frac{\pi}{2}-\omega}^{0}, \quad x \in X, \\
& \mathcal{P}_{q}(t) x=\int_{0}^{\infty} q \sigma \Psi_{q}(\sigma) T\left(\sigma t^{q}\right) x d \sigma, \quad t \in \frac{S_{\frac{\pi}{2}-\omega}^{0},}{2} \quad x \in X,
\end{aligned}
$$

and $\Psi_{q}(\sigma)$ is the function of Wright type such that

$$
\begin{aligned}
\Psi_{q}(z) & :=\sum_{n=0}^{\infty} \frac{(-z)^{n}}{n ! \Gamma(-q n+1-q)} \\
& =\frac{1}{\pi} \sum_{n=1}^{\infty} \frac{(-z)^{n}}{(n-1) !} \Gamma(n q) \sin (n \pi q), \quad z \in \mathrm{C},
\end{aligned}
$$

with $0<q<1$.

Remark 2.11 [[13], Remark 4.1] For every $x_{0} \in D\left(A^{\beta}\right)(\beta>1+\gamma)$, this mild solution (if any) is continuous at $t=0$.

Remark 2.12 [13] It is not difficult to verify that for $-1<r<\infty, \lambda>0$ and $-1<\alpha+\gamma$ $<0$,

(1) $\Psi_{q}(t) \geq 0, t>0$;

(2) $\int_{0}^{\infty} \Psi_{q}(t) t^{r} d t=\frac{\Gamma(1+r)}{\Gamma(1+q r)}$.

Then we have

$$
\begin{aligned}
& \left\|\mathcal{S}_{q}(t) x\right\| \leq \frac{C_{0} \Gamma(-\gamma)}{\Gamma(1-q(1+\gamma))} t^{-q(1+\gamma)}\|x\|, \\
& \left\|\mathcal{P}_{q}(t) x\right\| \leq \frac{q C_{0} \Gamma(1-\gamma)}{\Gamma(1-q \gamma)} t^{-q(1+\gamma)}\|x\|,
\end{aligned}
$$




$$
\begin{aligned}
\left\|A^{\alpha} \mathcal{P}_{q}(t) x\right\| & \leq\left\|\int_{0}^{\infty} q \sigma \Psi_{q}(\sigma) A^{\alpha} T\left(\sigma t^{q}\right) d \sigma\right\|\|x\| \\
& \leq q C^{\prime} \int_{0}^{\infty} \Psi_{q}(\sigma) t^{-q(\gamma+\alpha+1)} \sigma^{-\gamma-\alpha} d \sigma\|x\| \\
& \leq q C^{\prime} \frac{\Gamma(1-\gamma-\alpha)}{\Gamma(1-q(\gamma+\alpha))} t^{-q(\gamma+\alpha+1)}\|x\| .
\end{aligned}
$$

Lemma 2.13 [[13], Theorem 3.2] For $t>0,\left\{\mathcal{S}_{q}(t)\right\}$ and $\mathcal{P}_{q}(t)$ are continuous in the uniform operator topology.

Let

$$
X_{\alpha}=D\left(A^{\alpha}\right),
$$

and let $B C\left(\mathbf{R}_{+}, X_{\alpha}\right)$ denote the Banach space consisting of all real functions defined bounded and continuous from $\mathbf{R}_{+}$to $X_{\alpha}$ with the norm

$$
\|x\|_{\infty}=\sup _{t \in \mathbf{R}_{+}}\|x\|_{\alpha^{\prime}}
$$

for $x \in B C\left(\mathbf{R}_{+}, X_{\alpha}\right)$.

It is clear that $D\left(A^{\beta}\right) \subset D\left(A^{\alpha}\right)$.

Next, we present a measure of noncompactness introduced in [17].

For any nonempty and bounded subset $Y$ of the space $B C\left(\mathbf{R}_{+}, X\right)$ and a positive number $T$, we denote $\omega T(x, \varepsilon)$ as the modulus of continuity of function $x$ on the interval [0, $T$ ], where $x \in Y$ and $\varepsilon \geq 0$. Namely,

$$
\omega^{T}(x, \epsilon)=\sup \{\|x(t)-x(s)\| ; t, s \in[0, T],|t-s| \leq \epsilon\} .
$$

We then assume additionally

$$
\begin{aligned}
& \omega^{T}(Y, \epsilon)=\sup \left\{\omega^{T}(x, \epsilon) ; x \in Y\right\}, \\
& \omega_{0}^{T}(Y)=\lim _{\epsilon \rightarrow 0} \omega^{T}(Y, \epsilon), \\
& \omega_{0}(Y)=\lim _{T \rightarrow \infty} \omega_{0}^{T}(Y),
\end{aligned}
$$

and

$$
\operatorname{diam}(Y)=\sup \{\|x(t)-y(t)\| ; x, y \in Y\} .
$$

Finally, consider the function $\mu$ defined on the family $\mathcal{M}_{B C\left(\mathrm{R}_{+}, X\right)}$ by the formula:

$$
\mu(Y)=\omega_{0}(Y)+\limsup _{t \rightarrow \infty} \operatorname{diam}(Y) .
$$

It is known that $\mu$ is a measure of noncompactness.

Definition 2.14 The solution $x(t)$ of (1.1)-(1.2) is said to be globally attractive, if

$$
\lim _{t \rightarrow \infty}(x(t)-\gamma(t))=0,
$$

for any solution $y(t)$ of equation (1.1)-(1.2). 


\section{Main result}

In this section, we assume $-1<\alpha+\gamma<0$ and $0<\alpha<\beta<1$.

Theorem 3.1 Let $A \in \Theta_{\omega}^{\gamma}(X)$ and $0<\omega<\frac{\pi}{2}$. Assume that

(H1) $f: \mathbf{R}_{+} \times X_{\alpha} \rightarrow X$ is continuous, and there exists a positive function $v(\cdot): \mathbf{R}_{+} \rightarrow \mathbf{R}_{+}$ such that

$$
\begin{aligned}
& \left\{\begin{array}{l}
\|f(t, x)\| \leq v(t), \\
\text { the function } s \mapsto \frac{v(s)}{(t-s)^{1+q(\gamma+\alpha)}} \text { belongs to } L^{1}\left([0, t], \mathbf{R}_{+}\right),
\end{array}\right. \\
& \lim _{t \rightarrow \infty} \eta(t):=\lim _{t \rightarrow \infty} \int_{0}^{t} \frac{v(s)}{(t-s)^{1+q(\gamma+\alpha)}}=0 .
\end{aligned}
$$

(H2) The function $h \in B C\left(\mathbf{R}_{+}, X_{\alpha}\right)$ and there exists a constant $L \in(0,1)$ such that

$$
\left\|h\left(t_{1}, x\left(t_{1}\right)\right)-h\left(t_{2}, x\left(t_{2}\right)\right)\right\|_{\alpha} \leq L\left(\left|t_{1}-t_{2}\right|+\left\|x\left(t_{1}\right)-x\left(t_{2}\right)\right\|_{\alpha}\right) .
$$

(H3) For each nonempty, bounded set $D \subset B C\left(\mathbf{R}_{+}, X_{\alpha}\right)$, the family of functions

$$
\{t \rightarrow h(t, \varphi) ; \varphi \in D\}
$$

is equicontinuous.

Then

(1) for every $x_{0} \in D\left(A^{\beta}\right)$ with $\beta>1+\gamma$, the problem (1.1)-(1.2) has at least a mild solution on $B C\left(\mathbf{R}_{+}, X_{\alpha}\right)$;

(2) all solutions are globally attractive.

Proof. Consider the operator as follows:

$$
(H x)(t)=\mathcal{S}_{q}(t) x_{0}+h(t, x(t))+\int_{0}^{t}(t-s)^{q-1} \mathcal{P}_{q}(t-s) f(s, x(s)) d s, \quad t \geq 0 .
$$

Step 1: We prove that there exists a ball

$$
B_{r}=\left\{x \in B C\left(\mathbf{R}_{+}, X_{\alpha}\right) ;\|x\|_{\infty} \leq r\right\}
$$

with radius $r$ and centered at 0 , such that $H\left(B_{r}\right) \subset B_{r}$.

For any $r>0$ and $x \in B_{r}$, in view of (H2),

$$
\begin{aligned}
\|h(t, x(t))\|_{\alpha} & \leq\|h(t, x(t))-h(t, 0)\|_{\alpha}+\|h(t, 0)\|_{\alpha} \\
& \leq L r+M_{1}
\end{aligned}
$$

where

$$
M_{1}=\sup _{t \in \mathbf{R}_{+}}\|h(t, 0)\|_{\alpha} .
$$


By (3.2), we get

$$
\sup \{\eta(t)\} \leq K
$$

for a positive constant $K$.

Moreover, for arbitrary $x \in B_{r}$, by (2.3) and (3.1) we have

$$
\begin{aligned}
\|(H x)(t)\|_{\alpha} & \leq\left\|\mathcal{S}_{q}(t) x_{0}\right\|_{\alpha}+\|h(t, x(t))\|_{\alpha}+\int_{0}^{t}(t-s)^{q-1}\left\|\mathcal{P}_{q}(t-s) f(s, x(s))\right\|_{\alpha} d s \\
& \leq\left\|\mathcal{S}_{q}(t) x_{0}\right\|_{\alpha}+L r+M_{1}+q C^{\prime} \frac{\Gamma(1-\gamma-\alpha)}{\Gamma(1-q(\gamma+\alpha))} \int_{0}^{t}(t-s)^{-1-q(\gamma+\alpha)} v(s) d s \\
& \leq \sup _{t \in \mathbf{R}_{+}}\left\|\mathcal{S}_{q}(t) A^{\alpha} x_{0}\right\|+L r+M_{1}+q C^{\prime} \frac{\Gamma(1-\gamma-\alpha)}{\Gamma(1-q(\gamma+\alpha))} K
\end{aligned}
$$

Choose $r$ such that

$$
r \geq \frac{\sup _{t \in \mathbf{R}_{+}}\left\|\mathcal{S}_{q}(t) A^{\alpha} x_{0}\right\|+M_{1}+\frac{q C^{\prime} K \Gamma(1-\gamma-\alpha)}{\Gamma(1-q(\gamma+\alpha))}}{1-L} .
$$

Then

$$
\|(H x)(t)\|_{\alpha} \leq r
$$

that is $H\left(B_{r}\right) \subset B_{r}$.

Step 2: We prove that the operator $H$ is continuous on $B_{r}$.

Let $\left\{x_{n}\right\}$ be a sequence of $B_{r}$ such that $x_{n} \rightarrow \times$ in $B_{r}$ as $n \rightarrow \infty$. Then

$$
f\left(s, x_{n}(s)\right) \rightarrow f(s, x(s)), \text { as } n \rightarrow \infty
$$

since the function $f$ is continuous on $\mathbf{R}_{+} \times X_{\alpha}$.

For every $t \in[0, T]$, using (H2) and (2.3), we obtain

$$
\begin{aligned}
& \left\|\left(H x_{n}\right)(t)-(H x)(t)\right\|_{\alpha} \\
\leq & \left\|h\left(t, x_{n}(t)\right)-h(t, x(t))\right\|_{\alpha}+\left\|\int_{0}^{t}(t-s)^{q-1} \mathcal{P}_{q}(t-s)\left[f\left(s, x_{n}(s)\right)-f(s, x(s))\right] d s\right\|_{\alpha} \\
\leq & L\left\|x_{n}-x\right\|_{\infty}+\int_{0}^{t}(t-s)^{q-1}\left\|\mathcal{P}_{q}(t-s)\left[f\left(s, x_{n}(s)\right)-f(s, x(s))\right]\right\|_{\alpha} d s \\
\leq & L\left\|x_{n}-x\right\|_{\infty}+M_{2} \int_{0}^{t}(t-s)^{-1-q(\gamma+\alpha)}\left\|f\left(s, x_{n}(s)\right)-f(s, x(s))\right\| d s
\end{aligned}
$$

where

$$
M_{2}=q C^{\prime} \frac{\Gamma(1-\gamma-\alpha)}{\Gamma(1-q(\gamma+\alpha))} .
$$

Clearly, the first term of (3.5) tends to zero as $n \rightarrow \infty$. From the fact that

$$
\left\|f\left(s, x_{n}(s)\right)-f(s, x(s))\right\| \leq 2 v(s), \quad s \in \mathbf{R}_{+},
$$


(3.4), and the Lebesgue Dominated Convergence Theorem, it follows that the second term of (3.5) tends to zero too as $n \rightarrow \infty$.

Therefore, $H$ is continuous on $B_{r}$.

Step 3: Let $\Omega$ be arbitrary nonempty subset of $B_{r}$, we prove that

$$
\mu(H(\Omega)) \leq \mu(\Omega)
$$

Let us choose $x \in \Omega$ and $t_{1}, t_{2}$ with $\left|t_{2}-t_{1}\right|<\varepsilon$. Without loss of generality we may assume that $t_{1}<t_{2}$.

For any $T>0$, when $0=t_{1}<t_{2} \leq T$, we have

$$
\int_{0}^{t_{2}}\left\|\left(t_{2}-s\right)^{q-1} \mathcal{P}_{q}\left(t_{2}-s\right) f(s, x(s))\right\|_{\alpha} d s \leq M_{2} \int_{0}^{t_{2}}\left(t_{2}-s\right)^{-1-q(\gamma+\alpha)} v(s) d s .
$$

Hence $\left\|(H x)\left(t_{2}\right)\right\|$ is small as $t_{2}$ is small independently of $x \in \Omega$.

For $0<t_{1}<t_{2} \leq T$, taking into account our assumptions, we get

$$
\begin{aligned}
\left\|(H x)\left(t_{2}\right)-(H x)\left(t_{1}\right)\right\|_{\alpha} \leq & \left\|\left(\mathcal{S}_{q}\left(t_{2}\right)-\mathcal{S}_{q}\left(t_{1}\right)\right) x_{0}\right\|_{\alpha} \\
& +\left\|h\left(t_{2}, x\left(t_{2}\right)\right)-h\left(t_{1}, x\left(t_{1}\right)\right)\right\|_{\alpha} \\
& +\left\|\int_{0}^{t_{1}}\left[\left(t_{2}-s\right)^{q-1}-\left(t_{1}-s\right)^{q-1}\right] \mathcal{P}_{q}\left(t_{2}-s\right) f(s, x(s)) d s\right\|_{\alpha} \\
& +\left\|\int_{t_{1}}^{t_{2}}\left(t_{2}-s\right)^{q-1} \mathcal{P}_{q}\left(t_{2}-s\right) f(s, x(s)) d s\right\|_{\alpha} \\
& +\left\|\int_{0}^{t_{1}}\left(t_{1}-s\right)^{q-1}\left[\mathcal{P}_{q}\left(t_{2}-s\right)-\mathcal{P}_{q}\left(t_{1}-s\right)\right] f(s, x(s)) d s\right\|_{\alpha} \\
= & I_{1}+I_{2}+I_{3}+I_{4}+I_{5} .
\end{aligned}
$$

As a consequence of the continuity of $\left\{\mathcal{S}_{q}(t)\right\}$ in the uniform operator topology for $t$ $>0$, we know that

$$
I_{1} \rightarrow 0, \quad \text { as } t_{2} \rightarrow t_{1} .
$$

By $(\mathrm{H} 3)$, we see that

$$
I_{2} \rightarrow 0, \quad \text { as } t_{2} \rightarrow t_{1} .
$$

Using (2.3) and (H1), we have

$$
\begin{aligned}
I_{3} & =\left\|\int_{0}^{t_{1}}\left[\left(t_{2}-s\right)^{q-1}-\left(t_{1}-s\right)^{q-1}\right] \mathcal{P}_{q}\left(t_{2}-s\right) f(s, x(s)) d s\right\|_{\alpha} \\
& \leq q C^{\prime} \frac{\Gamma(1-\gamma-\alpha)}{\Gamma(1-q(\gamma+\alpha))} \int_{0}^{t_{1}} \frac{\left|\left(t_{2}-s\right)^{q-1}-\left(t_{1}-s\right)^{q-1}\right|}{\left(t_{2}-s\right)^{q-1}} \frac{v(s)}{\left(t_{2}-s\right)^{1+q(\gamma+\alpha)}} d s .
\end{aligned}
$$

Therefore, by (3.2), we get

$$
I_{3} \rightarrow 0, \quad \text { as } t_{2} \rightarrow t_{1} .
$$


Moreover, we have

$$
\begin{aligned}
I_{4} & =\left\|\int_{t_{1}}^{t_{2}}\left(t_{2}-s\right)^{q-1} \mathcal{P}_{q}\left(t_{2}-s\right) f(s, x(s)) d s\right\|_{\alpha} \\
& \leq q C^{\prime} \frac{\Gamma(1-\gamma-\alpha)}{\Gamma(1-q(\gamma+\alpha))} \int_{t_{1}}^{t_{2}}\left(t_{2}-s\right)^{-1-q(\gamma+\alpha)} \nu(s) d s \\
& \rightarrow 0, \text { as } t_{2} \rightarrow t_{1} .
\end{aligned}
$$

Finally, for $\varepsilon>0$ small enough, we obtain

$$
\begin{aligned}
& I_{5}=\left\|\int_{0}^{t_{1}}\left(t_{1}-s\right)^{q-1}\left[\mathcal{P}_{q}\left(t_{2}-s\right)-\mathcal{P}_{q}\left(t_{1}-s\right)\right] f(s, x(s)) d s\right\|_{\alpha} \\
& \leq q \int_{0}^{t_{1}} \int_{0}^{\infty} \sigma \Psi_{q}(\sigma)\left(t_{1}-s\right)^{q-1}\left\|T\left(\left(t_{2}-s\right)^{q} \sigma\right)-T\left(\left(t_{1}-s\right)^{q} \sigma\right)\right\|_{\alpha} \cdot v(s) d \sigma d s \\
& \leq q \int_{0}^{t_{1}-2 \varepsilon} \int_{0}^{\infty} \sigma \Psi_{q}(\sigma)\left(t_{1}-s\right)^{q-1}\left\|T\left(\left(t_{2}-s\right)^{q} \sigma-\varepsilon^{q} \sigma\right)-T\left(\left(t_{1}-s\right)^{q} \sigma-\varepsilon^{q} \sigma\right)\right\| \\
& \text { - }\left\|A^{\alpha} T\left(\varepsilon^{q} \sigma\right)\right\| v(s) d \sigma d s \\
& +M_{2} \int_{t_{1}-2 \varepsilon}^{t_{1}}\left(\frac{\left(t_{1}-s\right)^{q-1}}{\left(t_{1}-s\right)^{q(\alpha+\gamma+1)}}+\frac{\left(t_{1}-s\right)^{q-1}}{\left(t_{2}-s\right)^{q(\alpha+\gamma+1)}}\right) v(s) d s \\
& \leq \frac{q C^{\prime}}{\varepsilon^{q(\gamma+\alpha+1)}} \int_{0}^{t_{1}-2 \varepsilon} \int_{0}^{\infty} \sigma^{-\gamma-\alpha} \Psi_{q}(\sigma)\left\|T\left(\left(t_{2}-s\right)^{q} \sigma-\varepsilon^{q} \sigma\right)-T\left(\left(t_{1}-s\right)^{q} \sigma-\varepsilon^{q} \sigma\right)\right\| \\
& \frac{v(s)}{\left(t_{1}-s\right)^{1-q}} d \sigma d s \\
& +M_{2} \int_{t_{1}-2 \varepsilon}^{t_{1}}\left(\frac{\left(t_{1}-s\right)^{q-1}}{\left(t_{1}-s\right)^{q(\alpha+\gamma+1)}}+\frac{\left(t_{1}-s\right)^{q-1}}{\left(t_{2}-s\right)^{q(\alpha+\gamma+1)}}\right) v(s) d s \\
& =I_{5}^{\prime}+I_{5}^{\prime \prime} \text {. }
\end{aligned}
$$

The continuity of the function $t \rightarrow\|T(t)\| k$ for $t \in(0, T)$ implies that

$$
I_{5}^{\prime} \rightarrow 0, \quad \text { as } t_{2} \rightarrow t_{1} .
$$

Furthermore, it is easy to see that

$$
I_{5}^{\prime \prime} \rightarrow 0, \quad \text { as } \quad \varepsilon \rightarrow 0
$$

Thus, we obtain

$$
\omega_{0}^{T}(H \Omega)=0 .
$$

Consequently, we have

$$
\omega_{0}(H \Omega)=0 .
$$


Now, by our assumptions, for arbitrarily fixed $t \in \mathbf{R}_{+}$and $x, y \in \Omega$ we deduce that

$$
\begin{aligned}
& \|(H x)(t)-(H y)(t)\|_{\alpha} \\
\leq & \|h(t, x(t))-h(t, \gamma(t))\|_{\alpha}+\int_{0}^{t}(t-s)^{q-1}\left\|\mathcal{P}_{q}(t-s)[f(s, x(s))-f(s, y(s))]\right\|_{\alpha} d s \\
\leq & L\|x(t)-\gamma(t)\|_{\alpha}+2 M_{2} \eta(t) .
\end{aligned}
$$

By (3.2), we have

$$
\limsup _{t \rightarrow \infty} \operatorname{diam}(H \Omega)(t) \leq L \underset{t \rightarrow \infty}{\limsup } \operatorname{diam} \Omega(t) .
$$

Therefore, using the measure of noncompactness $\mu$ defined by the formula (2.4) and keeping in mind (3.7) and (3.8), we obtain

$$
\mu(H \Omega) \leq L \mu(\Omega)
$$

Step 4: We prove that the conclusion (1) is true.

Since $0<L<1$, in view of (3.9) and Lemma 2.5, we deduce that the operator $H$ has a fixed point $x$ in the ball $B_{r}$. Hence equation (1.1)-(1.2) has at least one mild solution $x$ $(t)$.

Step 5: We prove that the conclusion (2) is true.

Clearly, for any other mild solution $y(t)$ of Equation (1.1)-(1.2), we have

$$
\begin{aligned}
\|x(t)-y(t)\|_{\alpha} & =\|(H x)(t)-(H y)(t)\|_{\alpha} \\
& \leq L\|x(t)-\gamma(t)\|_{\alpha}+2 M_{2} \eta(t) .
\end{aligned}
$$

Then by (3.2) we have

$$
\lim _{t \rightarrow \infty}\|x(t)-\gamma(t)\|_{\alpha} \leq \frac{2 M_{2}}{1-L} \lim _{t \rightarrow \infty} \eta(t)=0 .
$$

That is, all mild solutions of (1.1)-(1.2) are globally attractive.

From the proof of Theorem 3.1, we can also see that the following theorem holds.

Theorem 3.2 Let $A \in \Theta_{\omega}^{\gamma}(X)$ and $0<\omega<\frac{\pi}{2}$. If the maps $f$ and $h$ satisfy

(H1) The function $f: \mathbf{R}_{+} \times X \rightarrow X$ is continuous, and there exists a positive function $v$ $(\cdot): \mathbf{R}_{+} \rightarrow \mathbf{R}_{+}$such that

$$
\begin{aligned}
& \|f(t, x)\| \leq v(t), \text { the function } s \mapsto \frac{v(s)}{(t-s)^{1+q \gamma}} \text { belongs to } L^{1}\left([0, t], \mathbf{R}_{+}\right), \\
& \lim _{t \rightarrow \infty} \eta(t):=\lim _{t \rightarrow \infty} \int_{0}^{t} \frac{v(s)}{(t-s)^{1+q \gamma}} d s=0 .
\end{aligned}
$$

(H2) The function $h \in B C\left(\mathbf{R}_{+}, X\right)$ and there exists a constant $L \in(0,1)$ such that

$$
\left\|h\left(t_{1}, x\left(t_{1}\right)\right)-h\left(t_{2}, x\left(t_{2}\right)\right)\right\| \leq L\left(\left|t_{1}-t_{2}\right|+\left\|x\left(t_{1}\right)-x\left(t_{2}\right)\right\|\right), \quad t_{1}, t_{2} \geq 0 .
$$

(H3) For each nonempty, bounded set $D \subset B C\left(\mathbf{R}_{+}, X\right)$, the family of functions

$$
\{t \rightarrow h(t, \varphi) ; \varphi \in D\}
$$

is equicontinuous. 
Then for every $x_{0} \in D\left(A^{\beta}\right)$ with $\beta>1+\gamma$, the problem (1.1)-(1.2) has at least a mild solution on $B C\left(\mathbf{R}_{+}, X\right)$ and all solutions are globally attractive.

\section{Applications}

Example 4.1: Let $\Omega$ be a bounded domain in $\mathbf{R}^{N}(N \geq 1)$ with boundary $\partial \Omega$ of class $C^{4}$. Let $X=C^{l}(\bar{\Omega})(0<l<1)$. Set

$$
\widetilde{A}=-\Delta, \quad D(\widetilde{A})=\left\{v \in C^{2+l}(\bar{\Omega}) ; v=0 \text { on } \partial \Omega\right\} .
$$

It follows from [[13], Example 1.2] that there exist $v, \varepsilon>0$ such that

$$
\tilde{A}+v \in \Theta_{\frac{\pi}{2}-\varepsilon}^{\gamma}\left(C^{l}(\bar{\Omega})\right), \quad \gamma=\frac{l}{2}-1 .
$$

We consider the fractional initial boundary value problem

$$
\left\{\begin{array}{l}
\frac{\partial^{q}}{\partial t^{q}}[u(t, x)-h(t, u(t, x))]=\Delta[u(t, x)-h(t, u(t, x))]+f(t, u(t, x)), \quad x \in \Omega, \\
\left.(u-h)\right|_{\partial \Omega}=0, \\
u(0, x)=u_{0}(x), \quad x \in \Omega,
\end{array}\right.
$$

where

$$
\begin{aligned}
& h(t, u(t, x))=\arctan t \cdot \tilde{A}^{-\alpha} \sin \left(1+\left|\tilde{A}^{\alpha} u(t, x)\right|\right) \int_{0}^{\infty} \frac{\zeta(t)}{1+|u(t, x)|} d t, \\
& f(t, u(t, x))=\left(t+r_{0}\right)^{a} \cdot \frac{|u(t, x)|}{1+|u(t, x)|}
\end{aligned}
$$

here $t>0, r_{0}$ is a positive constant,

$$
\frac{l}{2}<\alpha<1, \quad 0<\alpha+\frac{l}{2}<1, \quad-1<a<q\left(\alpha+\frac{l}{2}-1\right),
$$

$\zeta(\cdot) \in L^{1}\left(\mathbf{R}_{+}, \mathbf{R}\right)$ and $\frac{\pi}{2} \int_{0}^{\infty}|\zeta(t)| d t \leq L<1$.

The problem (4.1) can be written abstractly as (1.1)-(1.2).

Moreover, for $t \geq 0$, we can see

$$
\|f(t, u(t))\| \leq v(t),
$$

where $v(t):=\left(t+r_{0}\right)^{a}$.

It is clear that the function $s \rightarrow \frac{v(s)}{(t-s)^{1+q(\gamma+\alpha)}}$ belongs to $L^{1}\left([0, t], \mathbf{R}_{+}\right)$and

$$
\begin{aligned}
\int_{0}^{t} \frac{v(s)}{(t-s)^{1+q(\gamma+\alpha)}} d s & \leq \int_{0}^{t} \frac{s^{a}}{(t-s)^{1+q(\gamma+\alpha)}} d s \\
& =t^{a-q(\gamma+\alpha)} \int_{0}^{1} s^{a}(1-s)^{-1-q(\gamma+\alpha)} d s \\
& =t^{a-q(\gamma+\alpha)} B(a+1,-q(\gamma+\alpha)) \\
& \rightarrow 0, \quad t \rightarrow \infty
\end{aligned}
$$


where $B(\cdot, \cdot)$ is the Beta function.

Moreover, for $t_{1}, t_{2} \geq 0$ we have

$$
\begin{aligned}
& \left\|h\left(t_{1}, u\left(t_{1}\right)\right)-h\left(t_{2}, u\left(t_{2}\right)\right)\right\|_{\alpha} \\
= & \| \mid \arctan t_{1} \cdot \sin \left(1+\left|\tilde{A}^{\alpha} u\left(t_{1}, x\right)\right|\right) \int_{0}^{\infty} \frac{\zeta(t)}{1+|u(t, x)|} d t \\
& -\arctan t_{2} \cdot \sin \left(1+\left|\tilde{A}^{\alpha} u\left(t_{2}, x\right)\right|\right) \int_{0}^{\infty} \frac{\zeta(t)}{1+|u(t, x)|} d t \| \\
\leq & {\left[\left|\arctan t_{1}-\arctan t_{2}\right| \cdot\left\|\sin \left(1+\left|\tilde{A}^{\alpha} u\left(t_{1}, x\right)\right|\right)\right\|\right.} \\
& \left.+\left|\arctan t_{2}\right| \cdot\left\|\sin \left(1+\left|\tilde{A}^{\alpha} u\left(t_{1}, x\right)\right|\right)-\sin \left(1+\left|\tilde{A}^{\alpha} u\left(t_{2}, x\right)\right|\right)\right\|\right] \cdot \int_{0}^{\infty}|\zeta(t)| d t \\
\leq & {\left[\left|t_{1}-t_{2}\right|+\frac{\pi}{2}\left\|u\left(t_{1}\right)-u\left(t_{2}\right)\right\|_{\alpha}\right] \cdot \int_{0}^{\infty}|\zeta(t)| d t } \\
\leq & L\left(\left|t_{1}-t_{2}\right|+\left\|u\left(t_{1}\right)-u\left(t_{2}\right)\right\|_{\alpha}\right) .
\end{aligned}
$$

Consequently, it follows from Theorem 3.1 that, for every $u_{0} \in D\left(\tilde{A}^{\alpha+\beta}\right)$ with $1>\beta>\alpha>\frac{l}{2}$, the Equation (4.1) has at least a mild solution on $B C\left(\mathbf{R}_{+}, X_{\alpha}\right)$ and all solutions are globally attractive.

For example, if we put

$$
l=\frac{1}{12}, \quad \alpha=\frac{1}{8}, \quad a=-\frac{8}{9}, \quad q=\frac{1}{2}, \quad \zeta(t)=e^{-\pi t},
$$

then the assumptions can be satisfied.

Example 4.2: Let

$$
\widehat{A}=(-i \Delta+\sigma)^{\frac{1}{2}}, \quad D(\widehat{A})=W^{1,3}\left(\mathbf{R}^{2}\right) \quad \text { (a Sobolev space) },
$$

where $i \Delta$ is the Schro" dinger operator, $\sigma>0$ is a suitable constant.

Then $i \Delta$ generates a $\tilde{\beta}$-times integrated semigroup $S^{\tilde{\beta}}(t)$ with $\widetilde{\beta}=\frac{5}{12}$ on $L^{3}\left(\mathbf{R}^{2}\right)$ such that

$$
\left\|S^{\tilde{\beta}}(t)\right\|_{L\left(L^{3}\left(\mathbf{R}^{2}\right)\right)} \leq \widehat{M} t^{\tilde{\beta}}
$$

for all $t \geq 0$ and some constant $\widehat{M}>0$ (see [20]). Therefore, by virtue of [[21], Theorem 1.3.5 (P. 15)], [[21], Definition 1.3.1 (P. 12)] for $C=I$, we deduce that the operator $i \Delta+\sigma$ belongs to $\Theta_{\frac{\pi}{2}}^{\tilde{\beta}-1}\left(L^{3}\left(\mathbf{R}^{2}\right)\right)$, which denotes the family of all linear closed operators $A: D(A) \subset L^{3}\left(\mathbf{R}^{2}\right) \rightarrow L^{3}\left(\mathbf{R}^{2}\right)$ satisfying

$$
\sigma(A) \subset S_{\frac{\pi}{2}}=\left\{z \in \mathbf{C} \backslash\{0\} ;|\arg z| \leq \frac{\pi}{2}\right\} \cup\{0\},
$$


and for every $\frac{\pi}{2}<\mu<\pi$ there exists a constant $C \mu$ such that

$$
\|R(z ; A)\| \leq C_{\mu}|z|^{\tilde{\beta}-1}
$$

for all $z \in \mathbf{C} \backslash S_{\mu}$. Thus, it follows from [[19], Proposition 3.6] that $\widehat{A} \in \Theta_{\omega}^{\gamma}\left(L^{3}\left(\mathbf{R}^{2}\right)\right)$ for some $0<\omega<\frac{\pi}{2}$, where

$$
\gamma=-1+2 \tilde{\beta}=-\frac{1}{6}
$$

Let $X=L^{3}\left(\mathbf{R}^{2}\right)$, we consider the following equation:

$$
\left\{\begin{array}{c}
\frac{\partial^{q}}{\partial t^{q}}\left[u(t, x)-\sin t \cdot e^{-(1+|u(t, x)|)} \int_{0}^{\infty} k(t) \frac{|u(t, x)|}{1+|u(t, x)|} d t\right]= \\
-\widehat{A}\left[u(t, x)-\sin t \cdot e^{-(1+|u(t, x)|)} \int_{0}^{\infty} k(t) \frac{|u(t, x)|}{1+|u(t, x)|} d t\right. \\
+(t+1)^{b} \cdot \cos (1+|u(t, x)|), \quad x \in \mathbf{R}^{2},
\end{array}\right.
$$

where $t>0,-1<b<q \gamma$ and $k(\cdot) \in L^{1}\left(\mathbf{R}_{+}, \mathbf{R}\right)$ and $\int_{0}^{\infty}|k(t) d t| \leq L<1$.

Set

$$
\begin{aligned}
u(t)(x) & =u(t, x), \\
h(t, u(t))(x) & =\sin t \cdot e^{-(1+|u(t, x)|)} \int_{0}^{\infty} k(t) \frac{|u(t, x)|}{1+|u(t, x)|} d t, \\
f(t, u(t))(x) & =(t+1)^{b} \cdot \cos (1+|u(t, x)|) .
\end{aligned}
$$

Then the above Equation (4.2) can be reformulated as the abstract (1.1)-(1.2).

Moreover, for $t \geq 0$, we can see

$$
\|f(t, u(t))\| \leq v(t)
$$

where $v(t):=(t+1)^{b}$.

It is clear that the function $s \rightarrow \frac{v(s)}{(t-s)^{1+q \gamma}}$ belongs to $L^{1}\left([0, t], \mathbf{R}_{+}\right)$and

$$
\begin{aligned}
\int_{0}^{t} \frac{v(s)}{(t-s)^{1+q \gamma}} d s & \leq \int_{0}^{t} \frac{s^{b}}{(t-s)^{1+q \gamma}} d s \\
& =t^{b-q \gamma} \int_{0}^{1} s^{b}(1-s)^{-1-q \gamma} d s \\
& =t^{b-q \gamma} B(b+1,-q \gamma) \\
& \rightarrow 0, \quad t \rightarrow \infty .
\end{aligned}
$$


Moreover, for $t_{1}, t_{2} \geq 0$ we have

$$
\begin{aligned}
& \left\|h\left(t_{1}, u\left(t_{1}\right)\right)-h\left(t_{2}, u\left(t_{2}\right)\right)\right\| \\
& =\| \sin t_{1} \cdot e^{-\left(1+\left|u\left(t_{1}, x\right)\right|\right)} \int_{0}^{\infty} k(t) \frac{|u(t, x)|}{1+|u(t, x)|} d t \\
& -\sin t_{2} \cdot e^{-\left(1+\left|u\left(t_{2}, x\right)\right|\right)} \int_{0}^{\infty} k(t) \frac{|u(t, x)|}{1+|u(t, x)|} d t \mid \\
& \leq\left[\left|\sin t_{1}-\sin t_{2}\right| \cdot\left\|e^{-\left(1+\left|u\left(t_{1}, x\right)\right|\right)}\right\|\right. \\
& \left.+\left|\sin t_{2}\right| \cdot\left\|e^{-\left(1+\left|u\left(t_{1}, x\right)\right|\right)}-e^{-\left(1+\left|u\left(t_{2}, x\right)\right|\right)}\right\|\right] \cdot \int_{0}^{\infty}|k(t)| d t \\
& \leq\left[\left|t_{1}-t_{2}\right|+\left\|u\left(t_{1}\right)-u\left(t_{2}\right)\right\|\right] \cdot \int_{0}^{\infty}|k(t)| d t \\
& \leq L\left(\left|t_{1}-t_{2}\right|+\left\|u\left(t_{1}\right)-u\left(t_{2}\right)\right\|\right) .
\end{aligned}
$$

Consequently, it follows from Theorem 3.2 that, for every $u_{0} \in D\left(\widehat{A}^{\beta}\right)$ with $1>\beta>\frac{5}{6}$, the Equation (4.2) has at least a mild solution on $B C\left(\mathbf{R}_{+}, X\right)$ and all solutions are globally attractive.

For example, if we put

$$
q=\frac{1}{2}, \quad b=-\frac{1}{2}, \quad k(t)=e^{-2 t},
$$

then the assumptions can be satisfied.

\section{Acknowledgements}

The authors would like to thank the referees for helpful suggestions. The work was supported by the NSF of China (11171210).

\section{Author details}

${ }^{1}$ Department of Mathematics, Shanghai Jiao Tong University, Shanghai 200240, P. R. China ${ }^{2}$ School of Mathematics, Yunnan Normal University, Kunming 650092, P. R. China ${ }^{3}$ Texas A \& M University at Qatar, c/o Qatar Foundation, P. O. Box 5825, Doha, Qatar

\section{Authors' contributions}

$J \mathrm{~L}$ made the main contribution to Theorem 3.1 and Example 4.2, and drafted the manuscript. S-HY participated in the writing of Section 1 and made contributions to give some estimates in the proof of Theorem 3.1. FL made the main contribution to Example 4.1 and participated in giving the list of references. T-WH participated in the study of Example 4.2 and Definition 2.10. All authors read and approved the final manuscript.

\section{Competing interests}

The authors declare that they have no competing interests.

Received: 29 January 2012 Accepted: 4 April 2012 Published: 4 April 2012

\section{References}

1. Băleanu, D, Mustafa, OG, Agarwal, RP: Asymptotically linear solutions for some linear fractional differential equations. Abstr Appl Anal 2010, 8 (2010). (Article ID 865139)

2. Cuevas, C, de Souza, JC: S-asymptotically -periodic solutions of semilinear fractional integro-differential equations. Appl Math Lett. 22, 865-870 (2009). doi:10.1016/j.aml.2008.07.013

3. Cuevas, C, Lizama, C: Almost automorphic solutions to a class of semilinear fractional differential equations. Appl Math Lett. 21, 1315-1319 (2008). doi:10.1016/j.aml.2008.02.001

4. Diagana, T, Mophou, GM, NGuérékata, GM: On the existence of mild solutions to some semilinear fractional integrodifferential equations. Electron J Qual Theory Diff Equ. 58, 1-17 (2010) 
5. El-Borai, MM, Amar, D: On some fractional integro-differential equations with analytic semigroups. Int J Contemp Math Sci. 4, 1361-1371 (2009)

6. El-Borai, MM, El-Said El-Nadi, K, El-Akabawy, EG: On some fractional evolution equations. Comput Math Appl. 59(3):1352-1355 (2010). doi:10.1016/j.camwa.2009.05.005

7. Henderson, J, Ouahab, A: Impulsive differential inclusions with fractional order. Comput Math Appl. 59, 1191-1226 (2010). doi:10.1016/j.camwa.2009.05.011

8. Li, F: Mild solutions for fractional differential equations with nonlocal conditions. Adv Diff Equ 2010, 9 (2010). (Article ID 287861)

9. Lv, ZW, Liang, J, Xiao, TJ: Solutions to fractional differential equations with nonlocal initial condition in Banach spaces. Adv Diff Equ. 2010, 10 (2010)

10. Mophou, GM: Optimal control of fractional diffusion equation. Comput Math Appl. 61, 68-78 (2011). doi:10.1016/j. camwa.2010.10.030

11. Mophou, GM, N'Guérékata, GM: On some classes of almost automorphic functions and applications to fractional differential equations. Comput Math Appl. 59, 1310-1317 (2010). doi:10.1016/j.camwa.2009.05.008

12. Podlubny, I: Fractional Differential Equations. Academic Press, San Diego (1999)

13. Wang, RN, Chen, DH, Xiao, TJ: Abstract fravtional Cauchy problems with almost sectorial operators. J Diff Equ. 252, 202-235 (2012). doi:10.1016/j.jde.2011.08.048

14. Liang, J, Xiao, TJ: Semilinear integrodifferential equations with nonlocal initial conditions. Comp Math Appl. 47(67):863-875 (2004). doi:10.1016/50898-1221(04)90071-5

15. Pazy, A: Semigroups of Linear Operators and Applications to Partial Differential Equations, vol. 44 of Applied Mathematical Sciences. Springer, New York, NY, USA (1983)

16. von Wahl, W: Gebrochene potenzen eines elliptischen operators und parabolische diffren-tialgleichungen in Räuumen hölderstetiger Funktionen. Nachr Akad Wiss Göttingen, Math Phys Klasse. 11, $231-258$ (1972)

17. Banaś, J, Goebel, K: Measures of Noncompactness in Banach space, Lecture Notes in Pure and Applied Mathematics, Dekker, New York60 (1980)

18. Carvalho, AN, Dlotko, T, Nescimento, MJD: Nonautonomous semilinear evolution equations with almost sectorial operators. J Evol Equ. 8, 631-659 (2008). doi:10.1007/s00028-008-0394-3

19. Periago, F, Stadub, B: A functional calculus for almost sectorial operators and applications to abstract evolution equations. J Evol Equ. 2, 41-68 (2002). doi:10.1007/s00028-002-8079-9

20. van Neerven, JMAM, Straub, B: On the existence and growth of mild solutions of the abstract Cauchy problem for operators with polynomially bounded resolvent. Houston J Math. 24, 137-171 (1998)

21. Xiao, TJ, Liang, J: The Cauchy Problem for Higher Order Abstract Differential Equations. In Lecture Notes in Math, vol. 1701,Springer, Berlin, New York (1998)

doi:10.1186/1687-1847-2012-40

Cite this article as: Liang et al:: On the existence of mild solutions to the Cauchy problem for a class of fractional evolution equations. Advances in Difference Equations 2012 2012:40.

\section{Submit your manuscript to a SpringerOpen ${ }^{\circ}$ journal and benefit from:}

- Convenient online submission

- Rigorous peer review

- Immediate publication on acceptance

- Open access: articles freely available online

- High visibility within the field

- Retaining the copyright to your article

Submit your next manuscript at $\gg$ springeropen.com 